\title{
Surface Modification of Multiwall Carbon Nanotubes with Cationic Conjugated Polyelectrolytes: Fundamental Interactions and Intercalation into Conductive Poly(methyl-methacrylate) Composites
}

\author{
Alaa Ezzeddine, Zhuo Chen, Kirk S. Schanze, and Niveen M Khashab
}

ACS Appl. Mater. Interfaces, Just Accepted Manuscript • DOI: 10.1021/acsami.5b02540 • Publication Date (Web): 22 May 2015

Downloaded from http://pubs.acs.org on May 25, 2015

\section{Just Accepted}

"Just Accepted" manuscripts have been peer-reviewed and accepted for publication. They are posted online prior to technical editing, formatting for publication and author proofing. The American Chemical Society provides "Just Accepted" as a free service to the research community to expedite the dissemination of scientific material as soon as possible after acceptance. "Just Accepted" manuscripts appear in full in PDF format accompanied by an HTML abstract. "Just Accepted" manuscripts have been fully peer reviewed, but should not be considered the official version of record. They are accessible to all readers and citable by the Digital Object Identifier (DOI®). "Just Accepted" is an optional service offered to authors. Therefore, the "Just Accepted" Web site may not include all articles that will be published in the journal. After a manuscript is technically edited and formatted, it will be removed from the "Just Accepted" Web site and published as an ASAP article. Note that technical editing may introduce minor changes to the manuscript text and/or graphics which could affect content, and all legal disclaimers and ethical guidelines that apply to the journal pertain. ACS cannot be held responsible for errors or consequences arising from the use of information contained in these "Just Accepted" manuscripts. 


\title{
Surface Modification of Multiwall Carbon
}

\author{
Nanotubes with Cationic Conjugated
}

Polyelectrolytes: Fundamental Interactions and

Intercalation into Conductive Poly(methyl-

methacrylate) Composites

\author{
Alaa Ezzeddine, ${ }^{\dagger}$ Zhuo Chen, ${ }^{\dagger, \neq}$ Kirk S. Schanze, ${ }^{* \neq}$ and Niveen M. Khashab ${ }^{*, \dagger}$ \\ $\uparrow$ Smart Hybrid Materials (SHMs) Lab, Advanced Membranes and Porous Materials (AMPM) \\ Center, King Abdullah University of Science and Technology (KAUST), Thuwal 23955-6900, \\ Kingdom of Saudi Arabia \\ ₹ Department of Chemistry and Center for Macromolecular Science and Engineering, University \\ of Florida, P.O. Box 117200, Gainesville, Florida 32611-7200, United States
}

KEYWORDS: multiwall carbon nanotubes (MWCNTs), conjugated polyelectrolytes (CPEs), poly(methyl methacrylate) (PMMA), dispersion, electrical conductivity, composites 


\begin{abstract}
This research investigates the modification and dispersion and of pristine multiwalled carbon nanotubes (MWCNTs) through a simple solution mixing technique based on noncovalent interactions between poly(phenylene ethynylene) based conjugated polyelectrolytes functionalized with cationic imidazolium solubilizing groups (PIM-2 and PIM-4) and MWCNTs. Spectroscopic studies demonstrated the ability of PIMs to strongly interact with and efficiently disperse MWCNTs in different solvents mainly due to $\pi$-interactions between the PIMs and MWCNTs. Transmission electron microscopy and atomic force microscopy revealed the coating of the polyelectrolytes on the walls of the nanotubes. Scanning electron microscopy (SEM) studies confirm the homogenous dispersion of PIM modified MWCNTs in poly(methyl methacrylate) (PMMA) matrix. The addition of $1 \mathrm{wt} \%$ PIM modified MWCNTs to the matrix has led to a significant decrease in DC resistivity of the composite (13 orders of magnitude). The increase in electrical conductivity and the improvement in thermal and mechanical properties of the membranes containing the PIM modified MWCNTs is ascribed to the formation of MWCNTs networks and cross-linking sites that provided channels for the electrons to move in throughout the matrix and reinforced the interface between MWCNTs and PMMA.
\end{abstract}




\section{Introduction}

With a unique combination of electrical, mechanical and thermal properties, carbon nanotubes (CNTs) have drawn significant attention since their discovery in $1991 .^{1-5}$ CNTs can act as excellent nanofillers in the field of polymer-nanocomposites. ${ }^{6-9}$ In the last few years, interest in polymer/CNT composites has grown significantly due to their numerous applications including use in passive and active electronic and optoelectronic materials and devices. ${ }^{10-11}$ Polymer/CNT composites combine the good processability of polymers and the unique electrical, mechanical and thermal properties of CNTs, affording low-cost polymer materials with improved properties. ${ }^{12-14}$

Nevertheless, real-world applications of CNTs still face challenges. A key challenge to application is the poor miscibility between polymers and CNTs. ${ }^{15-16}$ Due to van der Waals attraction and $\pi-\pi$ stacking, CNTs are thermodynamically driven to form aggregates and clusters. The weak interfacial interaction between CNTs and polymers makes CNTs immiscible in the polymer phase, which decreases the improvement of the polymer properties dramatically. ${ }^{16-22}$ To overcome these problems, nanotubes were segregated out of their aggregated bundles by utilizing either covalent or non-covalent functionalization to modify their surface properties. ${ }^{23-26}$

Chemical functionalization of CNTs involves covalent attachment of functional groups (often polymer chains) to the open-end and sidewalls. This covalent functionalization of CNTs can improve CNT dispersion in solution and polymer matrices. Furthermore, covalent functionalization can also be used to modify and control the CNT/polymer interface for composite properties by varying the chemical functionality. ${ }^{27-29}$ However, a notable drawback of covalent functionalization is disruption of the extended $\pi$-conjugation in the nanotubes, thus affecting their electronic properties. 
By contrast, when CNTs are non-covalently functionalized, their structural integrity is preserved and consequently their properties are not disrupted, which is essential for their postsynthetic applications. ${ }^{30-32}$ Non-covalent functionalization involves utilizing specific molecules or polymers that adsorb onto the CNT surface to separate the CNT aggregates, and improve the dispersion of CNTs in solution or a polymer matrix. A library of established non-covalent functionalizing agents exists in the literature. Previous studies have examined the effect of low molecular weight molecules, surfactants and polymers including chitosan, sodium dodecyl sulfate (SDS), sodium cholate and conjugated polmers. ${ }^{33-36}$ Among these agents, conjugated polymers exhibit some of the best dispersing and solubilizing properties for CNTs. The strong $\pi$ $\pi$ interaction between conjugated polyarylenes and CNTs allows the backbone of these polymers to wrap CNTs efficiently. ${ }^{37-39}$ Bargen et al. suggested that wrapping of poly [p-2,5-bis(3propoxysulfonicacidsodiumsalt) $\}$ phenylene]ethynylene (PPES) around SWCNTs is not solely due to $\pi-\pi$ interaction between the conjugated backbone and the CNTs. They suggest that it also arises from the presence of the propoxysulfonate side-chains that interact with the nanotubes and the solvent resulting in helical wrapping of the polymer around nanotubes. ${ }^{40}$ Examples also include poly(3,4-ethylenedioxythiophene)-/poly(styrenesulfonate) (PEDOT/PSS) that adsorbs onto the surface of multiwall carbon nanotubes (MWCNTs) thus improving the dispersion and electrical properties of the nanocomposite. ${ }^{41}$ A hallmark study by Deria et al. showed that poly[2,6-1,5-bis(3-propoxysulfonic acid sodium salt)\} naphthylene]ethynylene (PNES) is able to helically wrap SWCNT with a periodic and constant morphology when a phase transfer catalyst such as 18-crown-6 is used. ${ }^{42}$ Our group has also reported poly-(9,9-dioctyfluorenyl-2,7-diyl) (PFO) to disperse multiwall CNTs in polyetherimide (PEI) matrix. The electrical conductivity of PEI was dramatically increased by the PFO/CNT dispersion. ${ }^{43}$ 
Ionic liquids have also been demonstrated to be good dispersion agents for CNTs via non-covalent functionalization. In a recent study, we reported a simple and scalable method for dispersing MWCNTs in a polyetherimide (PEI) matrix using an imidazolium based ionic liquid (IL). The strong interactions between the CNTs and the IL led to the formation of "bucky gels", which when dispersed within PEI yield films with excellent conductivity and thermal stability. ${ }^{44}$

Herein, we seek to combine the excellent dispersion capability of conjugated polymers and imidazolium solubilizing groups. Specifically, a set of conjugated polyelectrolytes (CPEs) featuring imidazolium side groups have been designed and prepared (Figure 1). The design of these polymers takes into consideration the idea that the poly(phenylene ethynylene) backbone will adsorb onto with the CNT surfaces, while the pendant imidazole groups will act as solubilizing and stabilizing units, giving rise to the ability to disperse the modified CNTs in solvents and polymer matrices. We characterize the interaction between the CPEs and MWCNTs by using optical spectroscopy and electron and atomic force microscopy, and the electrical, thermal and mechanical properties of PMMA composites with the CPE modified CNTs are then explored. The results show that the imidazole conjugated poylelectrolytes serve as excellent solubilizing and dispersing agents for MWCNTs, giving rise to composites that exhibit moderate DC conductivity and improved thermal and mechanical properties.

\section{Experimental Methods}

Materials. PMMA in fine powdered was supplied by SABIC Innovative Plastics TM (average $M_{w} \sim 990000$ ). MWCNTs were purchased from Sigma Aldrich with a purity $>95 \%$. The diameter of the MWCNTs used is 6-9 nm and the length is 2-5 $\mu \mathrm{m}$. Dimethylformamide (DMF) was purchased from Sigma Aldrich and used as received. The conjugated polyelectrolytes PIM-2 and PIM-4 were prepared and characterized as described elsewhere. ${ }^{45}$ 
Preparation of Conjugated Polyelectrolyte Coated MWCNTs. PIM-2 or PIM-4 were dissolved in (DMF) under bath type sonication for 90 minutes followed by stirring for 12 hours at room temperature to obtain a homogenous pale yellow solution. Pristine MWCNTs were mixed with the PIM/DMF solution then sonicated for 90 minutes to form a homogenous suspension. Mixtures of different concentrations of PIM/MWCNT were prepared and studied.

Preparation of the PMMA/PIM/MWCNTs Nanocomposites. PMMA was dissolved in DMF and allowed to stir at room temperature for one hour until a clear solution was obtained. PMMA was then added to the PIM/MWCNT solutions to obtain different mixtures of PMMA/PIM-MWCNT. The PMMA/PIM-MWCNT dispersions were then sonicated for one hour and then stirred for 12 hours. The mixtures were then cast into a glass petri dish followed by solvent evaporation at $50{ }^{\circ} \mathrm{C}$ in a vacuum oven for 24 hours to obtain the desired polymer nanocomposite membrane. The resulting membranes were $0.1 \mathrm{~mm}$ thick, and they were heated at $70{ }^{\circ} \mathrm{C}$ for 24 hours eliminate any remaining solvent.

Instrumentation and Methods. Ultraviolet-visible absorption spectra were completed in DMF solutions using Varian Cary 5000 UV-VIS-NIR absorption spectrometer at room temperature. Fluorescence spectra were measured using Varian Cary Eclipse fluorescence spectrometer.

High Resolution Transmission Electron Microscopy (HRTEM) images were obtained using a Titan CT (FEI Company) operating at $300 \mathrm{kV}$ and equipped with a $4 \mathrm{k}$ x $4 \mathrm{k}$ CCD camera (Gatan). Standard (TEM) images were collected using a Tecnai G2 Spirit TWIN, a 20$120 \mathrm{kV} / \mathrm{LaB}$. The PIM/MWCNT solution was drop casted on a lacy copper grid stabilized with carbon (300 MESH, EMS) and dried for 6 hours in a desiccator prior to the analysis. Atomic Force Microscopy (AFM) data were obtained by depositing the sample on a freshly 
cleaved Mica surface using an Agilent 5400 SPM instrument (USA). Scanning electron microscopy (SEM) images were taken using FEI Nova Nano 630 FEG HR-SEM equipped with a Through the Lens Detector (TLD) (USA). In addition, the samples were cryofractured and mounted vertically for cross-sectional imaging.

Dynamic mechanical analysis (DMA) was conducted using a DMA 242C (Netzsch, Germany) in the thin tension mode, at a constant frequency of $1 \mathrm{~Hz}$, static force at $0.5 \mathrm{~N}$, dynamic force at $1 \mathrm{~N}$, heating rate of $2 \mathrm{k} / \mathrm{min}$ under air atmosphere and temperature range of 30 to $160{ }^{\circ} \mathrm{C}$.

The decomposition behavior of the composites was studied using a Thermogravimetric Analyzer (TGA) TG 209 F1 Iris (Netzsch, Germany) under nitrogen from 30 to $500{ }^{\circ} \mathrm{C}$, with a heating rate of $10{ }^{\circ} \mathrm{C} / \mathrm{min}$. The thermal behavior of the nanocomposites was studied using a Differential Scanning Calorimeter (DSC) (DSC 204 F1 Phoenix, Netzsch, Germany). The heating rate was $10{ }^{\circ} \mathrm{C} / \mathrm{min}$ under a nitrogen atmosphere with a flow rate of $40 \mathrm{ml} / \mathrm{min}$.

Four Point Probe form MDC (CMT-SR2000N) was used to measure samples with resistivity lower than $10^{5} \Omega . \mathrm{cm}$. For the composites with higher resistivity, a constant voltage of $100 \mathrm{~V}$ DC was applied across the specimen using a Keithley model 248 high voltage supply (USA). The current was monitored with a Keithley 6517B electrometer equipped with an 8009 test fixture (Keithley Instruments Inc.). The samples had a diameter of $8 \mathrm{~cm}$ and a thickness of $0.1 \mathrm{~mm}$. The average value of three measurements is obtained for each different nanocomposite. Finally, the Zeta Potential was measured using Zetasizer Nano ZS from Malvern.

\section{Results and Discussion}

Study and Characterization of CPEs/MWCNTs Dispersion. Two conjugated polyelectrolytes were used in the present investigation as shown in Figure 1 (PIM-2 and PIM-4). 
These polymers feature a poly(phenylene ethynylene) (PPE) conjugated backbone that is functionalized with alkyl imidazolium side groups. The polymers differ in the ratio of imidazolium to the phenylene ethynylene (PE) repeats: PIM-2 features two imidzaolium units for every two PE units, whereas PIM-4 features two imidazolium units on every PE repeat. As a result of this difference, PIM-4 features a higher charge density on the PPE backbone compared to PIM-2. Both of the polymers are blue-violet absorbing and they emit strong blue-green fluorescence. ${ }^{46}$ PIM-2 and PIM-4 are easily dissolved in water, alcohols and polar organic solvents and, interestingly it is found that when their solutions are mixed in various ratios with MWCNTs they are able to disperse the nanotubes in various organic and aqueous solvents. For example, as shown in Figure 2a, the dispersions of PIM-2/MWCNTs in DMF solvent are homogenous, with no evidence of MWCNT precipitation observed after more than 8 months, indicating the high stability of the dispersion.

In order to probe the physiochemical interactions between the imidazole CPEs and the MWCNTs, we used optical spectroscopy to examine changes that occur when the species are mixed. ${ }^{47-48}$ Thus, the UV-visible absorption spectra were recorded for PIM-2 (22 $\left.\mu \mathrm{g} / \mathrm{ml}\right)$ in DMF as reference solution, and for PIM-2/MWCNTs dispersions with varying concentrations of the MWCNTs (Figure 2b). The major absorption band observed for PIM-2 has $\lambda_{\max } \sim 417 \mathrm{~nm}$, which is characteristic of the PPE backbone. ${ }^{49}$ With increased MWCNT concentration, the overall absorption increases due to the broad visible absorption by the nanotubes; the absorption peak due to PIM-2 is clearly visible, but becomes less resolved as the overall absorption increases above 2 absorbance units. ${ }^{50}$

Fluorescence spectroscopy was also used to probe the electronic interaction between PIM-2 and the MWCNTs. The fluorescence spectrum of PIM-2 exhibits a strong fluorescence 
emission with a band maximum at $\lambda_{\max }=450 \mathrm{~nm}$ (Figure $2 \mathrm{c}$ ). Upon addition of MWCNTs into the PIM-2 solution, the fluorescence is progressively quenched as the concentration of the MWCNTs is increased. More than $40 \%$ of PIM-2 fluorescence emission is quenched by MWCNTs for 1:1 mass ratio (MWCNT:PIM-2), and the emission is entirely quenched at a 6:1 mass ratio. This significant quenching of the PIM-2 fluorescence is likely due to either energy and/or electron transfer from PIM-2 to the MWCNTs. ${ }^{51-52}$ (In order to confirm that the fluorescence quenching is not due to self-absorption, the quenching experiment was repeated with optically dilute samples, see Fig. S5). The finding clearly indicates the presence of strong interactions between the CNTs and the PIM-2 backbone, and is also considered to suggest the existence of $\pi-\pi$ stacking between PIM-2 and the MWCNTs. ${ }^{53-54}$ Another important point to note is that the quenching trend in the fluorescence spectra obtained from our measurement is distinct to that typically seen arising from quenching of CPEs due to inter-chain aggregation (red-shift accompanying reduction in intensity), and thus further suggests the unique interaction between the MWCNTs and PIM-2.

Zeta potential measurements were used to characterize and compare PIM-2/MWCNT and PIM-4/MWCNT dispersions. This experiment relates the dispersion to the magnitude of electrostatic interactions between CPE modified MWCNTs. ${ }^{55-56}$ As seen in Figure S1, pristine MWCNTs are nonionic and their zeta potential is zero in neutral solutions. However, when the MWCNTs are coated with the polyionic CPE chains, they acquire a charge and therefore consequently display a surface potential where the sign reflects the charge on the polyelectrolyte. As seen in Figure S1, the zeta potential values are $+45 \mathrm{mV}$ for 4:1 MWCNT:PIM-2 (wt:wt) and +90 mV for 4:1 MWCNT:PIM-4 (wt:wt). The positive values are indicative that a positive potential is imparted to the MWCNTs as a result of adsorption of the imidazolium CPEs. ${ }^{57}$ 
Interestingly, the zeta potential of the PIM-4 coated MWCNTs is twice that of the PIM-2 coated MWCNTs. This increase in the potential is a result of the higher charge density on the PIM-4 backbone; the doubling of the zeta potential for PIM-4 suggests that the two polyelectrolytes adsorbs with similar surface coverage (based on the number of phenylene ethynylene repeat units) on the MWCNTs. The effect of the different surface potential imparted by PIM-2 and PIM-4 on the dispersion of the MWCNTs and the conductivity of the PMMA/PIM/MWCNTs composites is discussed later in this paper.

Transmission electron microscopy (TEM) was used to characterize the interaction between the CPEs and MWCNTs by real-space imaging of the morphology of the CPE-wrapped MWCNTs. Figure 3 compares the TEM images of pristine and CPE modified carbon nanotubes. First, comparison of the wide-field images (Fig. 3a-c) reveals the improved dispersion of the PIM-2 and PIM-4 modified MWCNTs compared to the pristine sample. Interestingly, the results also suggest that the PIM-4 modified sample is better dispersed compared to PIM-2 (Fig. 3c vs. $3 b$, consistent with expectation based on the greater surface potential for PIM-4 modified sample. Figure 3d-f compares high-resolution TEM (HR-TEM) images of pristine and the PIM2 and PIM-4 modified MWCNTs. Here it is possible to visualize what appears to be a disordered, continuous thin coating layer (1-3 nm) on the surface of the CPE modified MWCNTs (Figure 3e and f) as compared to surface morphology of the pristine sample, which appears distinct and smooth (Figure 3d). ${ }^{52,58}$

Finally, the size and surface morphology of the PIM-2 modified MWCNTs were characterized using atomic force microscopy (AFM). ${ }^{59-60}$ The AFM images were obtained on PIM-2/MWCNTs dispersed in DMF and then deposited onto a mica substrate. Figure 4 shows the AFM image of the sample, along with several plots of the z-axis height profile for specific 
regions. The z-axis scans collected in Figure $4 \mathrm{~b}$ show that the diameter of PIM-2/MWCNT falls in the range 9-14 $\mathrm{nm}$, which is $3-5 \mathrm{~nm}$ greater than the original pristine MWCNTs $(6-9 \mathrm{~nm}$ as reported by the supplier). Moreover, the z-axis scan shown in Figure 4c which was taken along the surface of a single nanotube reveals an irregular height profile, consistent with the notion that PIM-2 exists on the nanotube surfaces as a continuous, but irregular coating. Note that this morphology is distinct from the "helical" wrapping that has been reported for wrapping of single-walled CNTs by a helical CPE by Therien and co-workers. ${ }^{37-38,40}$

Morphology of PMMA/PIM-2/MWCNTs Nanocomposites. Composites consisting of PIM-2/MWCNTs within a poly(methyl methacrylate) matrix (PMMA) were fabricated by using a solution casting method where PMMA was mixed and sonicated with the previously modified PIM-2/MWCNTs followed by casting into a glass petri dish. The objective of this work is to obtain a uniform dispersion of the PIM-2/MWCNTs within the PMMA matrix, leading to enhanced properties of the PMMA composite (e.g., conductivity, mechanical strength, and thermal stability).

High resolution scanning electron microscopy (HR-SEM) was used to image the dispersion of the PIM-2 modified- and pristine MWCNTs within a PMMA matrix (Figure 5). The morphology of a cryofractured PMMA nanocomposites sample with 1 wt $\%$ pristine MWCNT is shown in Figs. 5b and c. By comparison Figs. 5d and e show a cryofractured PMMA composite that contains a dispersion of PIM-2/MWCNT ( 1 wt- $\%$ each relative to PMMA, 1:1 wt-ratio PIM-2/MWCNT). As a point of reference, Fig. 5a indicates that the cross sectional morphology of 1-wt\% PIM-2 in PMMA is smooth and flat with no obvious phase separation. First, as seen in Figs. $5 \mathrm{~b}$ and $\mathrm{c}$, the addition of $1 \%$ of the non-modified MWCNTs to PMMA results in a clear deformation in the fractured surface of the PMMA composite with 
observable phase separation (see arrows highlighted in Figure 5b). It is evident that there is relatively poor dispersion of the pristine MWCNTs in the PMMA phase, as the number of dispersed nanotubes within the polymer matrix is relatively low. Moreover, a closer inspection of the structure of the pristine MWCNT/PMMA composite clearly reveals the presence of MWCNT aggregates (see circle highlighted in Figure 5c). By comparison, upon dispersing PIM2 modified MWCNTs within the PMMA matrix, an obvious enhancement in the nanotube dispersion within the polymer matrix is noticed (Figure 5d). The images show good uniformity and dispersity of the modified MWCNTs as compared to the composites with the pristine MWCNTs. Moreover, almost no phase separation is observed, and as a result a homogenous surface is obtained. Moreover, Figure 5e shows a number of contacts between the adjacent nanotubes in the composite matrix. This contact could facilitate electron transport, enhancing the overall electrical conductivity of the composites, as discussed below.

Conductivity of PMMA/PIM-2/MWCNTs Composites. A series of experiments were performed to explore the DC resistivity of PMMA/MWCNT composites. Four Point Probe was used to measure the samples with resistivity lower than $10^{5} \Omega . \mathrm{cm}$ while a Keithley model 248 was used for samples with higher resistivity. The studied samples had a diameter of $8 \mathrm{~cm}$ and a thickness of $0.1 \mathrm{~mm}$. The average value of three different measurements was obtained for each nanocomposite. These experiments were carried out using samples that contained a fixed amount of PIM-2 or PIM-4 relative to PMMA (0.25 - 1 wt-\%) and varying loading of MWCNT (0 - 2 wt-\%). The results of these measurements are compiled in Figure 6, which illustrates the variation of the DC resistivity as a function of MWCNT wt- $\%$ for different PIM loadings. Note that in the process of forming the composites with pristine MWCNTs, the unmodified MWCNTs tend to aggregate in the polymer matrix due to their substantial van der Waal interactions (Figure 
S3). Because of the MWCNT aggregation, the MWCNTs network is discontinuous within the PMMA matrix and consequently, very low conductivity was observed.

As expected, the volume resistivity of pristine PMMA is very high $\left(10^{16} \Omega . \mathrm{cm}\right)$ which is consistent with its insulating properties. Upon the addition of $1 \mathrm{wt} \%$ PIM-2 only to the PMMA sheet (Figure 6a), the volume resistivity decreased slightly indicating that no substantial conductivity is added to the PMMA matrix in the presence of PIM-2 alone. However, the resistivity decreased sharply with the increasing concentration of the PIM-2 modified MWCNTs. For $0.25 \mathrm{wt} \%$ MWCNTs, the resistivity was measured as $6 \times 10^{13} \Omega . \mathrm{cm}$ compared to the value for pristine PMMA which was $10^{16} \Omega . \mathrm{cm}$. This high resistivity value is explained as being due to the fact that the nanotube concentration within the PMMA matrix is still quite low (and below the percolation threshold). We rationalize that the MWCNTs are possibly segregated from each other in the polymer matrix which limits the electron transfer/transport between the carbon nanotubes and results in only minimal enhancement in conductivity. By comparison, the addition of increased concentration of MWCNTs to the PMMA matrix resulted in a pronounced reduction in volume resistivity of PMMA to $10^{11} \Omega . \mathrm{cm}$ for $0.5 \mathrm{wt} \%$ MWCNTs and $1.46 \times 10^{5} \Omega . \mathrm{cm}$ for 1 wt $\%$ MWCNTs, likely due to formation of a percolation network. The resistivity reached a minimum at $2 \mathrm{wt} \%$ loading of the PIM-2 modified MWCNTs where the composite has a resistivity of $1.6 \times 10^{3} \Omega . c m$ compared to $10^{16} \Omega . \mathrm{cm}$ for the pristine PMMA. Overall, the outcome of dispersing PIM-2/MWCNTs in PMMA matrix with 1-wt\% PIM-2 is a dramatic enhancement in conductivity up to 13 orders of magnitude in comparison to the neat PMMA.

Upon decreasing the concentration of PIM-2 in the polymer matrix to $0.5 \mathrm{wt} \%$ (Figure $6 b)$, a similar effect on the conductivity of the composites was observed with a slight increase in the conductivity values obtained. Interestingly, when only $0.25 \mathrm{wt} \%$ PIM-2 was used, the 
conductivity was higher for all composites concentrations, and it reached a maximum value of 26 $\Omega . \mathrm{cm}$ for $2 \mathrm{wt} \%$ MWCNTs (Figure 6c). This enhancement maybe attributed to the lower coating of the PIM-2 on the walls of the CNTs. The presence of fewer charges on the modified MWCNTs likely leads to less repulsion between the MWCNTs giving rise to improved intertube contact and enhanced electron transfer between the nanotubes.

The DC resistivity of composites with $0.5 \mathrm{wt} \% \mathrm{PIM}-4$ relative to MWCNT was also examined (Figure 6d). For $1 \mathrm{wt} \%$ MWCNT content, the resistivity of the composite was higher compared to its similar PIM-2 composites, likely due to the fact that PIM-4 has higher charge density (highlighted by the zeta potential measurements, see above). The greater surface charge on the PIM-4 dispersed MWCNTs leads to an increase in the repulsion between the nanotubes (increase in the average inter-tube separation distance) and consequently less inter-tube contact and less electron transfer. On the other hand, the presence of higher concentration of MWCNTs (2-wt \%), the conductivity increases as a result of more CNTs interaction. Taken together, to the best of our knowledge, the results reported here for the PIM modified MWCNT/PMMA composites represent the lowest reported values of DC resistivity observed for MWCNT/PMMA composites at similar concentrations of MWCNTs. This clearly supports the notion that the PIM family of CPEs has remarkable properties with respect to their ability to wrap CNTs and stabilize their dispersions.

In previous work, Jian et al. has reported the modification of polycarbonate (PC) nanocomposites using (MWCNTs) coated with a thin layer of conductive (PEDOT/PSS). The resulted MWCNT/(PEDOT/PSS)/PC composite had a volume resistivity of $(4.2 \pm 1) \times 10^{9} \Omega . c m$ for $1 \mathrm{wt} \%$ loading of MWCNTs. ${ }^{41}$ In a similar manner, our lab has reported the use of PFO to disperse MWCNTs in PEI matrix. The resulted PFO/MWCNT/PEI composite in this case had a 
volume resistivity of $3 \times 10^{3} \Omega \cdot \mathrm{cm}$ at $2 \mathrm{wt} \%$ loading of MWCNTs and $0.5 \mathrm{wt} \%$ loading of PFO. $^{43}$

To demonstrate the potential application of the modified composite membrane in electrical devices, a PMMA/PIM-2/MWCNTs membrane with $2 \mathrm{wt} \%$ MWCNTs and $1 \mathrm{wt} \%$ PIM was demonstrated to act as a conductor, connected in series with circuit for a light emitting diode (60 mW, see Figure S4).

Thermal Analysis of the PMMA/PIM-2/MWCNTs Composites. To explore the effect of the PIM-2 modified MWCNTs on the thermal stability of the PMMA composites, the thermal properties of PMMA and the modified composites were studied using differential scanning calorimetry (DSC) and thermogravimetric analysis (TGA) under nitrogen atmosphere. Results are summarized in Figure 7 and Table 1, and in the supporting information. The importance of this study is to determine the dispersion state of the modified MWCNTs within the polymer matrix and the effect of any possible MWCNTs aggregates on the thermal behavior of PMMA.

Figure 7a compares DSC thermograms for PMMA composites with pristine MWCNTs and with PIM-2 modified MWCNTs. For all of the samples, a transition is observed at $\sim 120{ }^{\circ} \mathrm{C}$, which is analogous to the glass transition temperature $\left(\mathrm{T}_{\mathrm{g}}\right)$ of syndiotactic PMMA. ${ }^{61}$ Literature reports the $T_{g}$ of neat PMMA very widely depending on the degree of tacticity. ${ }^{61-62}$ A sample of the neat PMMA used herein exhibited a glass transition temperature, $\mathrm{T}_{\mathrm{g}} \sim 122{ }^{\circ} \mathrm{C}$. However, the presence of pristine MWCNTs in the composite results in a slightly lower glass transition temperature $\left(\mathrm{T}_{\mathrm{g}}=121{ }^{\circ} \mathrm{C}\right)$ compared to neat PMMA. This can be ascribed to the multiwalled carbon nanotubes that are typically aggregates having a small influence on the $\mathrm{T}_{\mathrm{g}}$. By contrast, the presence of PIM-2 modified MWCNTs in the PMMA matrix leads to a slight but systematic increase in the $\mathrm{T}_{\mathrm{g}}$ of the nanocomposites. For $1 \mathrm{wt} \%$ of PIM-2/MWCNTs, the $\mathrm{T}_{\mathrm{g}}$ increased $2{ }^{\circ} \mathrm{C}$ 
compared to the neat PMMA. Interestingly, these findings that the PIM-2/MWCNTs lead to a slight increase in the $T_{g}$ are in concert with findings that dispersion of polymer grafted graphene in PMMA leads to slight increase in the $\mathrm{T}_{\mathrm{g}}{ }^{63}$ The effect is attributed to the "attractive" interaction between the graphene surface and the PMMA matrix, which decreases the mobility of the chains in proximity to the interface. It is likely that a similar effect arises in the PIM2/PMMA composites, which points to the fact that the PIM is a good interface stabilizing agent, having a favorable interaction with the PMMA matrix.

The thermal gravimetric analyses for the nanocomposites in comparison with the neat PMMA matrix were carried out in order to examine their thermal stability. Figure S2 and Table 1 list the TGA results obtained for PMMA and composites with different concentrations of MWCNTs, while Figure $7 \mathrm{~b}$ gives a summary presentation. A clear difference is noticed in the thermal stability of the nanocomposites with varying concentration of the nanotubes, and in general, the modified composites exhibited enhanced stability compared to neat PMMA. The differential thermogravimetric data (DTGA) presented in Figure 7b shows two degradation steps for neat PMMA, but only a single step in the presence of MWCNTs. The first step (around 280 ${ }^{\circ} \mathrm{C}$ ) is related to bond scission at unsaturated ends, and is caused by loss of $-\mathrm{CO}_{2} \mathrm{CH}_{3}$ groups, while the second step (at $361{ }^{\circ} \mathrm{C}$ ) is due to arbitrary cleavage within the polymer backbone. In the presence of MWCNTs, only the second weight loss is observed, for reasons that are not entirely clear. $^{64}$

The differential thermograms exhibit an overall increase in the decomposition temperature of the nanocomposites with $1 \mathrm{wt} \% \mathrm{PIM}-2 / \mathrm{MWCNTs}$, which maximizes at $379{ }^{\circ} \mathrm{C}$. The temperature is slightly lower for the composite with 1wt \% pristine MWCNTs, which decomposes at $366{ }^{\circ} \mathrm{C}$, and the neat PMMA which decomposes at $361{ }^{\circ} \mathrm{C}$. The presence of a 
single weight loss step and the increase in the decomposition temperature of the MWCNT modified nanocomposites can be explained as a result of the interaction between the MWCNTs and the polymer. The presence of MWCNTs gives rise to restricted motion and results in less segmental mobility of PMMA chains. The decrease in the mobility helps in hindering chain scission and increases the thermal stability of the polymer.

Moreover, PMMA exhibited $10 \mathrm{wt} \%$ weight loss at $245{ }^{\circ} \mathrm{C}$ and this temperature gradually increased with the increasing concentration of PIM-2/MWCNTs until it reached its maximum of $295{ }^{\circ} \mathrm{C}$ for $1 \mathrm{wt} \%$ PIM-2/MWCNTs. At $\mathrm{T}_{30}$ (where the material loses $30 \%$ of weight) the temperature difference reached $55{ }^{\circ} \mathrm{C}$ between the neat PMMA and the $1 \mathrm{wt} \% \mathrm{PIM}-2 / \mathrm{MWCNTs}$ modified composite (Table 1). It is also observed that the composite with the pristine MWCNTs exhibited a lower thermal stability at all temperatures compared to the composite having PIM2/MWCNTs for the same concentration.

Overall, the results reveal that the presence of cross-linked PIM-2/MWCNTs complex structure and the robust PIM/MWCNT/PMMA networks can support the thermal stability of resulted composites. This improvement is possibly due to the excellent dispersion of the nanotubes in the PMMA matrix which in turn has strengthened the interfacial interactions between the carbon nanotubes and PMMA. The effect of additives on the thermal properties of polymers is broadly studied in the literature. For example, in a recent study by Goncalves et al. PMMA was grafted on graphene oxide to give GPMMA. The GPMMA nanosheets where then used as a filler to enhance the properties of PMMA films. The resultant PMMA/GPMMA composites have shown increase in the thermal stability of the polymer thin films compared to the composite with the non-modified GO. ${ }^{65}$ 


\section{Dynamic Mechanical Analysis of PIM-2/MWCNTs PMMA Composites. Dynamic} mechanical analysis (DMA) is an essential tool for studying the effect of additives on the mechanical properties of a polymer composite. ${ }^{66}$ In the present study, DMA was applied to determine the storage modulus (E') as a function of temperature for neat PMMA as well as the MWCNT composites, Figure 8. The results clearly indicate an increase in the storage modulus of PMMA upon the addition of un-modified MWCNT. An even greater increase in storage modulus is seen for the composite that contains PIM-2/MWCNTs at low temperatures (up to 90 $\left.{ }^{\circ} \mathrm{C}\right)$. The noticeable enhancement in the storage modulus can be attributed to excellent dispersion of PIM-2 modified MWCNTs and to the development many physical cross-linking sites that reinforced the interface between MWCNTs and PMMA. At higher temperatures, the polymer chains of PMMA/PIM-2/MWCNTs composite are more flexible than that of PMMA/MWCNTs which led to a lower storage modulus. This softening in the polymer chains can be attributed to the good miscibility between PIM-2 and PMMA.

\section{Conclusion}

This study clearly reveals that the imidazole functionalized poly(phenylene ethynylene) polyelectrolytes interact strongly with MWCNTs, as confirmed by fluorescence quenching, zeta potential, TEM and AFM. The strong interaction between the CPE chains and the CNTs likely arises from $\pi-\pi$ (dispersion) interaction between the phenylene ethynylene backbone and the CNT surface, with the imidazole units acting as solubilizing groups which also separate the individual nanotubes due to charge repulsion. As a result of these favorable effects, the PIM modified MWCNTs are easily dispersed in solution as well as into a polymer matrix, as exemplified herein by PMMA. The resulting PIM/MWCNT/PMMA composites exhibit excellent DC conductivity as well as improved thermal and mechanical properties. Compared to 
other surface active molecules and polymers that have been used as dispersants for CNTs, ${ }^{32,}$ 67-68 we believe that the results presented here demonstrate that imidazole conjugated polyelectrolytes are among the leading materials for solubilizing and dispersing carbon nanotubes.

\title{
Associated content
}

\section{Supporting Information}

Zeta potential records comparing the effect of PIM-2 and PIM-4 on the surface charge of the MWCNTs; TGA curves of PMMA, PMMA composites with $1 \mathrm{wt} \%$ PIM-2 and various concentrations of MWCNTs, PMMA composite with $1 \mathrm{wt} \%$ pristine MWCNTs; summary of the composition of all studied composites.

This material is available free of charge via the internet at http://pubs.acs.org.

\author{
Author information \\ * Corresponding Authors \\ Niveen M. Khashab \\ e-mail: niveen.khashab@kaust.edu.sa, TEL:+966-128082410 \\ Kirk S. Schanze \\ e-mail: kschanze@,chem.ufl.edu, TEL: 352-392-9133

\section{Acknowledgement}

This work was generously supported by King Abdullah University of Science and Technology (KAUST).

\section{References:}

(1) Zhang, D. H.; Ryu, K.; Liu, X. L.; Polikarpov, E.; Ly, J.; Tompson, M. E.; Zhou, C. W., Transparent, Conductive, and Flexible Carbon Nanotube Films and Their Application in Organic Light-Emitting Diodes. Nano Lett. 2006, 6, 1880-1886.

(2) Rosario-Canales, M. R.; Deria, P.; Therien, M. J.; Santiago-Aviles, J. J., Composite Electronic Materials Based on Poly(3,4-Propylenedioxythiophene) and Highly Charged 
Poly(Aryleneethynylene)-Wrapped Carbon Nanotubes for Supercapacitors. ACS Appl. Mater. Interfaces. 2012, 4, 102-109.

(3) Cui, H.; Hong, C.; Ying, A.; Yang, X.; Ren, S., Ultrathin Gold Nanowire-Functionalized Carbon Nanotubes for Hybrid Molecular Sensing. ACS Nano 2013, 7, 7805-7811.

(4) Cho, D.-Y.; Eun, K.; Choa, S.-H.; Kim, H.-K., Highly Flexible and Stretchable Carbon Nanotube Network Electrodes Prepared by Simple Brush Painting for Cost-Effective Flexible Organic Solar Cells. Carbon 2014, 66, 530-538.

(5) Wang, H.; Koleilat Ghada, I.; Liu, P.; Jimenez-Oses, G.; Lai, Y.-C.; Vosgueritchian, M.; Fang, Y.; Park, S.; Houk Kendall, N.; Bao, Z., High-Yield Sorting of Small-Diameter Carbon Nanotubes for Solar Cells and Transistors. ACS Nano 2014, 8, 2609-2617.

(6) Lee, M.; Jeon, H.; Min, B. H.; Kim, J. H., Morphology and Electrical Properties of Polymethyl Methacrylate/Poly(Styrene-Co-Acrylonitrile)/Multi-Walled Carbon Nanotube Nanocomposites. J. Appl. Polym. Sci. 2011, 121, 743-749.

(7) Logakis, E.; Pandis, C.; Pissis, P.; Pionteck, J.; Poetschke, P., Highly Conducting Poly(Methyl Methacrylate)/Carbon Nanotubes Composites: Investigation on Their Thermal, Dynamic-Mechanical, Electrical and Dielectric Properties. Compos. Sci. Technol. 2011, 71, 854862.

(8) Paul, A.; Grady, B. P.; Ford, W. T., Pmma Composites of Single-Walled Carbon NanotubesGraft-Pmma. J. Appl. Polym. Sci. 2014, 131, 39884-39895.

(9) Tang, B.; Wu, C.; Lin, T.; Zhang, S., Heat-Resistant Pmma Photonic Crystal Films with Bright Structural Color. Dyes Pigm. 2013, 99, 1022-1028.

(10) Pang, H.; Bao, Y.; Xu, L.; Yan, D.-X.; Zhang, W.-Q.; Wang, J.-H.; Li, Z.-M., DoubleSegregated Carbon Nanotube-Polymer Conductive Composites as Candidates for Liquid Sensing Materials. J. Mater. Chem. A 2013, 1, 4177-4181.

(11) Yamada, T.; Hayamizu, Y.; Yamamoto, Y.; Yomogida, Y.; Izadi-Najafabadi, A.; Futaba, D. N.; Hata, K., A Stretchable Carbon Nanotube Strain Sensor for Human-Motion Detection. Nat. Nanotechnol. 2011, 6, 296-301. 
(12) Sun, X.; Zhang, Z.; Lu, X.; Guan, G.; Li, H.; Peng, H., Electric Current Test Paper Based on Conjugated Polymers and Aligned Carbon Nanotubes. Angew. Chem., Int. Ed. Engl. 2013, 52, 7776-7780.

(13) Kumar, S.; Li, B.; Caceres, S.; Maguire, R. G.; Zhong, W.-H., Dramatic Property Enhancement in Polyetherimide Using Low-Cost Commercially Functionalized Multi-Walled Carbon Nanotubes Via a Facile Solution Processing Method. Nanotechnology 2009, 20, 465708465717.

(14) Kyoung-Yong Chun, Y. O., Jonghyun Rho, Jong-Hyun Ahn, Young-Jin Kim Hyouk Ryeol Choil and Seunghyun Baik, Highly Conductive, Printable and Stretchable Composite Films of Carbon Nanotubes and Silver. Nat. Nanotechnol. 2010, 5, 853-857.

(15) Huang, D.; Wang, A., Non-Covalently Functionalized Multiwalled Carbon Nanotubes by Chitosan and Their Synergistic Reinforcing Effects in Pva Films. RSC Advances 2013, 3, 12101216.

(16) Teng, N.-Y.; Dallmeyer, I.; Kadla, J. F., Effect of Softwood Kraft Lignin Fractionation on the Dispersion of Multiwalled Carbon Nanotubes. Ind. Eng. Chem. Res. 2013, 52, 6311-6317.

(17) Bosch, S.; Zeininger, L.; Hauke, F.; Hirsch, A., A Supramolecular Approach for the Facile Solubilization and Separation of Covalently Functionalized Single-Walled Carbon Nanotubes. Chem. - Eur. J. 2014, 20, 2537-2541.

(18) Massuyeau, F.; Zhao, Y.; Mel, A. A.; Yaya, A.; Geschier, F.; Gautron, E.; Lefrant, S.; Mevellec, J. Y.; Ewels, C.; Hsu, C.-S.; Faulques, E.; Wéry, J.; Duvail, J. L., Improved Photoconductive Properties of Composite Nanofibers Based on Aligned Conjugated Polymer and Single-Walled Carbon Nanotubes. Nano Res. 2013, 6, 149-158.

(19) Nish, A.; Hwang, J. Y.; Doig, J.; Nicholas, R. J., Highly Selective Dispersion of SingleWalled Carbon Nanotubes Using Aromatic Polymers. Nat. Nanotechnol. 2007, 2, 640-646.

(20) Strano, M. S., Nanocomposites: Polymer-Wrapped Nanotubes. Nat. Mater. 2006, 5, 433434.

(21) Baskaran, D.; Mays, J. W.; Bratcher, M. S., Noncovalent and Nonspecific Molecular Interactions of Polymers with Multiwalled Carbon Nanotubes. Chem. Mater. 2005, 17, 33893397. 
(22) Suhr, J.; Koratkar, N.; Keblinski, P.; Ajayan, P., Viscoelasticity in Carbon Nanotube Composites. Nat. Mater. 2005, 4, 134-137.

(23) Yamamoto, N., Guzman de Villoria, Roberto, Wardle, Brian L, Electrical and Thermal Property Enhancement of Fiber-Reinforced Polymer Laminate Composites through Controlled Implementation of Multi-Walled Carbon Nanotubes. Compos. Sci. Technol. 2012, 72, 20092015.

(24) Furmanchuk, A. o.; Leszczynski, J.; Tretiak, S.; Kilina, S. V., Morphology and Optical Response of Carbon Nanotubes Functionalized by Conjugated Polymers. J. Phys. Chem. C 2012, 116, 6831-6840.

(25) Etika, K. C.; Jochum, F. D.; Theato, P.; Grunlan, J. C., Temperature Controlled Dispersion of Carbon Nanotubes in Water with Pyrene-Functionalized Poly(N-Cyclopropylacrylamide). $J$. Am. Chem. Soc. 2009, 131, 13598-13599.

(26) Ge, J. J.; Zhang, D.; Li, Q.; Hou, H.; Graham, M. J.; Dai, L.; Harris, F. W.; Cheng, S. Z. D., Multiwalled Carbon Nanotubes with Chemically Grafted Polyetherimides. J. Am. Chem. Soc. 2005, 127, 9984-9985.

(27) Mildred Quintana, H. T., Anna Llanes-Pallas, Riccardo Marega Davide Bonifazi and Maurizio Prato, Multiple Hydrogen Bond Interactions in the Processing of Functionalized MultiWalled Carbon Nanotubes. ACS Nano 2012, 6, 23-31.

(28) Wang, F.; Swager, T. M., Diverse Chemiresistors Based Upon Covalently Modified Multiwalled Carbon Nanotubes. J. Am. Chem. Soc. 2011, 133, 11181-11193.

(29) Bahr, J. L.; Tour, J. M., Covalent Chemistry of Single-Wall Carbon Nanotubes. J. Mater. Chem. 2002, 12, 1952-1958.

(30) Sgobba, V.; Giancane, G.; Cannoletta, D.; Operamolla, A.; Hassan Omar, O.; Farinola, G. M.; Guldi, D. M.; Valli, L., Langmuir-Schaefer Films for Aligned Carbon Nanotubes Functionalized with a Conjugate Polymer and Photoelectrochemical Response Enhancement. ACS Appl. Mater. Interfaces. 2014, 6, 153-158.

(31) Yang, Z.; Xue, Z.; Liao, Y.; Zhou, X.; Zhou, J.; Zhu, J.; Xie, X., Hierarchical Hybrids of Carbon Nanotubes in Amphiphilic Poly(Ethylene Oxide)-Block-Polyaniline through a Facile Method: From Smooth to Thorny. Langmuir 2013, 29, 3757-3764. 
(32) Zhao, W.; Liu, Y. T.; Feng, Q. P.; Xie, X. M.; Wang, X. H.; Ye, X. Y., Dispersion and Noncovalent Modification of Multiwalled Carbon Nanotubes by Various Polystyrene-Based Polymers. J. Appl. Polym. Sci. 2008, 109, 3525-3532.

(33) Castillo, J. J.; Torres, M. H.; Molina, D. R.; Castillo-Leon, J.; Svendsen, W. E.; Escobar, P.; Martinez, F., Monitoring the Functionalization of Single-Walled Carbon Nanotubes with Chitosan and Folic Acid by Two-Dimensional Diffusion-Ordered Nmr Spectroscopy. Carbon 2012, 50, 2691-2697.

(34) Sun, Z.; Nicolosi, V.; Rickard, D.; Bergin, S. D.; Aherne, D.; Coleman, J. N., Quantitative Evaluation of Surfactant-Stabilized Single-Walled Carbon Nanotubes: Dispersion Quality and Its Correlation with Zeta Potential. J. Phys. Chem. C 2008, 112, 10692-10699.

(35) Ali-Boucetta, H.; Al-Jamal, K. T.; McCarthy, D.; Prato, M.; Bianco, A.; Kostarelos, K., Multiwalled Carbon Nanotube-Doxorubicin Supramolecular Complexes for Cancer Therapeutics. Chem. Commun. (Cambridge, U. K.) 2008, 459-461.

(36) Foroutan, M.; Nasrabadi, A. T., Investigation of the Interfacial Binding between SingleWalled Carbon Nanotubes and Heterocyclic Conjugated Polymers. J. Phys. Chem. B 2010, 114, 5320-5326.

(37) Kang, Y. K.; Lee, O.-S.; Deria, P.; Kim, S. H.; Park, T.-H.; Bonnell, D. A.; Saven, J. G.; Therien, M. J., Helical Wrapping of Single-Walled Carbon Nanotubes by Water Soluble Poly(PPhenyleneethynylene). Nano Lett. 2009, 9, 1414-1418.

(38) Deria, P.; Von Bargen, C. D.; Olivier, J. H.; Kumbhar, A. S.; Saven, J. G.; Therien, M. J., Single-Handed Helical Wrapping of Single-Walled Carbon Nanotubes by Chiral, Ionic, Semiconducting Polymers. J. Am. Chem. Soc. 2013, 135, 16220-16234.

(39) Samanta, S. K.; Fritsch, M.; Scherf, U.; Gomulya, W.; Bisri, S. Z.; Loi, M. A., Conjugated Polymer-Assisted Dispersion of Single-Wall Carbon Nanotubes: The Power of Polymer Wrapping. Acc. Chem. Res. 2014, 47, 2446-2456.

(40) Von Bargen, C. D.; MacDermaid, C. M.; Lee, O. S.; Deria, P.; Therien, M. J.; Saven, J. G., Origins of the Helical Wrapping of Phenyleneethynylene Polymers About Single-Walled Carbon Nanotubes. J. Phys. Chem. B 2013, 117, 12953-12965.

(41) Zhou, J.; Lubineau, G., Improving Electrical Conductivity in Polycarbonate Nanocomposites Using Highly Conductive Pedot/Pss Coated Mwcnts. ACS Appl. Mater. Interfaces. 2013, 5, 6189-6200. 
(42) Deria, P.; Sinks, L. E.; Park, T. H.; Tomezsko, D. M.; Brukman, M. J.; Bonnell, D. A.; Therien, M. J., Phase Transfer Catalysts Drive Diverse Organic Solvent Solubility of SingleWalled Carbon Nanotubes Helically Wrapped by Ionic, Semiconducting Polymers. Nano Lett. 2010, 10, 4192-4199.

(43) Chen, Y.; Tao, J.; Li, S.; Khashab, N. M., Compositing Polyetherimide with Polyfluorene Wrapped Carbon Nanotubes for Enhanced Interfacial Interaction and Conductivity. ACS Appl. Mater. Interfaces. 2014, 6, 9013-9022.

(44) Chen, Y.; Tao, J.; Deng, L.; Li, L.; Li, J.; Yang, Y.; Khashab, N. M., Polyetherimide/Bucky Gels Nanocomposites with Superior Conductivity and Thermal Stability. ACS Appl. Mater. Interfaces. 2013, 5, 7478-7484.

(45) Parthasarathy, A. P., H. C.; Hill, E. H.; Huang, Y.; Whitten, D. G.; Schanze, K. S., Conjugated Polyelectrolytes with Imidazolium Solubilizing Groups. Photophysics, Quenching and Application for to Photodynamic Inactivation of Bacteria. Submitted 2015.

(46) Koenen, J.-M.; Zhu, X.; Pan, Z.; Feng, F.; Yang, J.; Schanze, K. S., Enhanced Fluorescence Properties of Poly(Phenylene Ethynylene)-Conjugated Polyelectrolytes Designed to Avoid Aggregation. ACS Macro Lett. 2014, 3, 405-409.

(47) Peng, H.; Sun, X.; Cai, F.; Chen, X.; Zhu, Y.; Liao, G.; Chen, D.; Li, Q.; Lu, Y.; Zhu, Y.; Jia, Q., Electrochromatic Carbon Nanotube/Polydiacetylene Nanocomposite Fibres. Nat. Nanotechnol. 2009, 4, 738-741.

(48) Yi, W.; Malkovskiy, A.; Chu, Q.; Sokolov, A. P.; Lebron Colon, M.; Meador, M.; Pang, Y., Wrapping of Single-Walled Carbon Nanotubes by a П-Conjugated Polymer: The Role of Polymer Conformation-Controlled Size Selectivity. J. Phys. Chem. B 2008, 112, 12263-12269.

(49) Muller, J. G.; Anni, M.; Scherf, U.; Lupton, J. M.; Feldmann, J., Vibrational Fluorescence Spectroscopy of Single Conjugated Polymer Molecules. Phys. Rev. B: Condens. Matter Mater. Phys. 2004, 70, 035205-035210.

(50) Star, A.; Stoddart, J. F.; Steuerman, D.; Diehl, M.; Boukai, A.; Wong, E. W.; Yang, X.; Chung, S.-W.; Choi, H.; Heath, J. R., Preparation and Properties of Polymer-Wrapped SingleWalled Carbon Nanotubes. Angew. Chem., Int. Ed. Engl. 2001, 40, 1721-1725.

(51) Chen, J.; Liu, H.; Weimer, W. A.; Halls, M. D.; Waldeck, D. H.; Walker, G. C., Noncovalent Engineering of Carbon Nanotube Surfaces by Rigid, Functional Conjugated Polymers. J. Am. Chem. Soc. 2002, 124, 9034-9035. 
(52) Baykal, B.; Ibrahimova, V.; Er, G.; Bengu, E.; Tuncel, D., Dispersion of Multi-Walled Carbon Nanotubes in an Aqueous Medium by Water-Dispersible Conjugated Polymer Nanoparticles. Chem. Commun. (Cambridge, U. K.) 2010, 46, 6762-6764.

(53) Cheng, F. Y.; Imin, P.; Maunders, C.; Botton, G.; Adronov, A., Soluble, Discrete Supramolecular Complexes of Single-Walled Carbon Nanotubes with Fluorene-Based Conjugated Polymers. Macromolecules 2008, 41, 2304-2308.

(54) Yuan, W. Z.; Zhao, H.; Shen, X. Y.; Mahtab, F.; Lam, J. W. Y.; Sun, J. Z.; Tang, B. Z., Luminogenic Polyacetylenes and Conjugated Polyelectrolytes: Synthesis, Hybridization with Carbon Nanotubes, Aggregation-Induced Emission, Superamplification in Emission Quenching by Explosives, and Fluorescent Assay for Protein Quantitation. Macromolecules 2009, 42, 94009411.

(55) Brian White, S. B., Stephen O’Brien, Nicholas J. Turro ,Irving P. Herman, Zeta-Potential Measurements of Surfactant-Wrapped Individual Single-Walled Carbon Nanotubes. J. Phys. Chem. C 2007, 111, 13684-13690.

(56) Lotya, M.; Hernandez, Y.; King, P. J.; Smith, R. J.; Nicolosi, V.; Karlsson, L. S.; Blighe, F. M.; De, S.; Wang, Z.; McGovern, I. T.; Duesberg, G. S.; Coleman, J. N., Liquid Phase Production of Graphene by Exfoliation of Graphite in Surfactant/Water Solutions. J. Am. Chem. Soc. 2009, 131, 3611-3620.

(57) De la Cruz, E. F.; Zheng, Y. D.; Torres, E.; Li, W.; Song, W. H.; Burugapalli, K., Zeta Potential of Modified Multi-Walled Carbon Nanotubes in Presence of Poly (Vinyl Alcohol) Hydrogel. Int. J. Electrochem. Sci. 2012, 7, 3577-3590.

(58) Liu, J.; Moo-Young, J.; McInnis, M.; Pasquinelli, M. A.; Zhai, L., Conjugated Polymer Assemblies on Carbon Nanotubes. Macromolecules 2014, 47, 705-712.

(59) Bonhommeau, S.; Deria, P.; Glesner, M. G.; Talaga, D.; Najjar, S.; Belin, C.; Auneau, L.; Trainini, S.; Therien, M. J.; Rodriguez, V., Raman Spectroscopic Investigation of Individual Single-Walled Carbon Nanotubes Helically Wrapped by Ionic, Semiconducting Polymers. J. Phys. Chem. C 2013, 117, 14840-14849.

(60) Zhu, J.; Shim, B. S.; Di Prima, M.; Kotov, N. A., Transparent Conductors from Carbon Nanotubes Lbl-Assembled with Polymer Dopant with Pi-Pi Electron Transfer. J. Am. Chem. Soc. 2011, 133, 7450-7460. 
(61) Thomas, P.; Ravindran, R. S. E.; Varma, K. B. R., Structural, Thermal and Electrical Properties of Poly(Methyl Methacrylate)/Cacu3ti4o12 Composite Sheets Fabricated Via Melt Mixing. J. Therm. Anal. Calorim. 2014, 115, 1311-1319.

(62) Teng, H.; Koike, K.; Zhou, D.; Satoh, Z.; Koike, Y.; Okamoto, Y., High Glass Transition Temperatures of Poly(Methyl Methacrylate) Prepared by Free Radical Initiators. J. Polym. Sci., Part A: Polym. Chem. 2009, 47, 315-317.

(63) Liao, K.-H.; Kobayashi, S.; Kim, H.; Abdala, A. A.; Macosko, C. W., Influence of Functionalized Graphene Sheets on Modulus and Glass Transition of Pmma. Macromolecules 2014, 47, 7674-7676.

(64) Ferriol, M.; Gentilhomme, A.; Cochez, M.; Oget, N.; Mieloszynski, J. L., Thermal Degradation of Poly(Methyl Methacrylate) (Pmma): Modelling of Dtg and Tg Curves. Polym. Degrad. Stabil. 2003, 79, 271-281.

(65) Gonçalves, G.; Marques, P. A. A. P.; Barros-Timmons, A.; Bdkin, I.; Singh, M. K.; Emami, N.; Grácio, J., Graphene Oxide Modified with Pmma Via Atrp as a Reinforcement Filler. $J$. Mater. Chem. 2010, 20, 9927-9934.

(66) Wenge Zheng, S.-C. W., Electrical Conductivity and Dielectric Properties of Pmma/Expanded Graphite Composites. Compos. Sci. Technol. 2003, 63, 225-235.

(67) Lee, J.; Aida, T., "Bucky Gels" for Tailoring Electroactive Materials and Devices: The Composites of Carbon Materials with Ionic Liquids. Chem. Commun. (Cambridge, U. K.) 2011, 47, 6757-6762.

(68) Huang, Y. Y.; Terentjev, E. M., Dispersion of Carbon Nanotubes: Mixing, Sonication, Stabilization, and Composite Properties. Polymers 2012, 4, 275-295. 

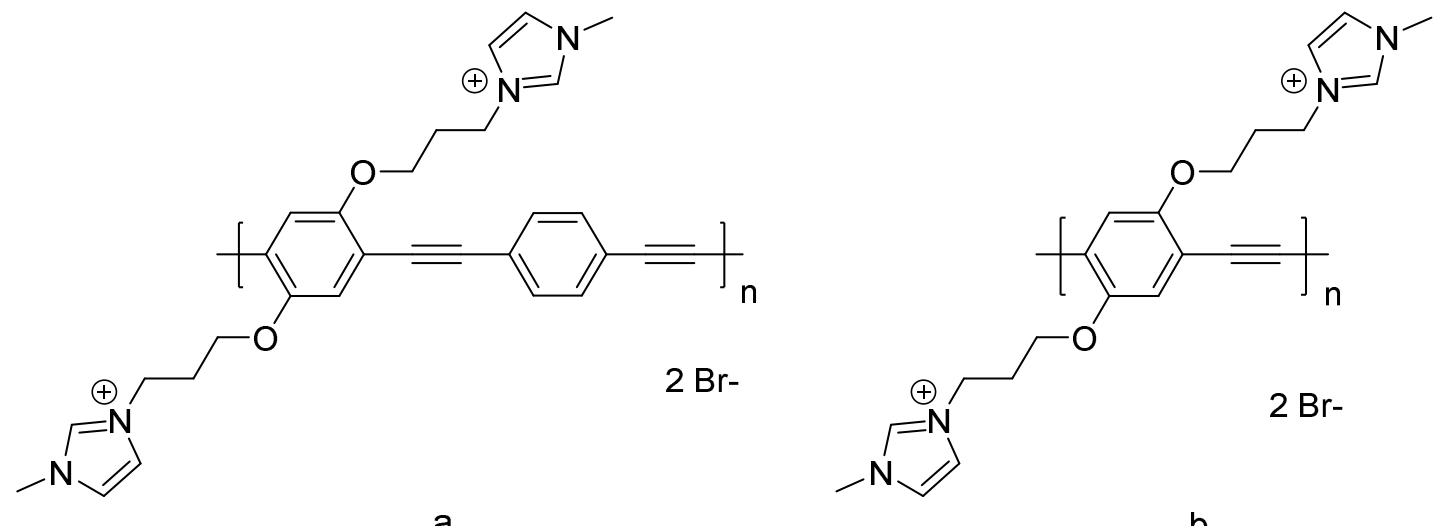<smiles>CCC(C)(C)C(=O)OC</smiles>

a

b

C

Figure 1 Chemical structure of (a) (PIM-2), (b) PIM-4, (c) poly(methyl methacrylate) (PMMA)

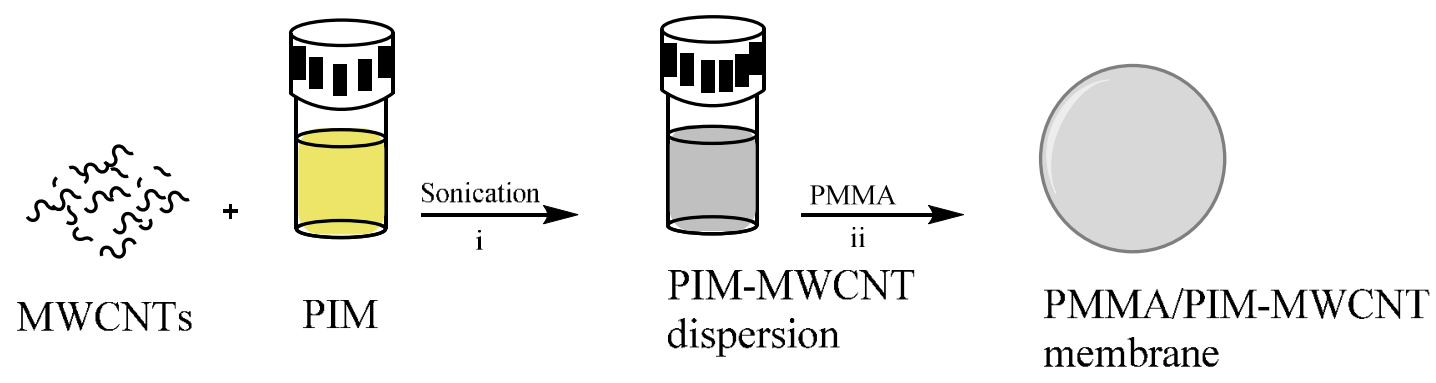

Scheme 1 Preparation of the PMMA/PIM-MWCNTs nanocomposites. (i) Sonication of PIM with MWCNTs for 90 minutes followed by stirring for 12 hours. (ii) Sonication of PMMA with the obtained PIM-MWCNTs dispersion for 1 hour followed by stirring for 12 hours. 
(a) a $\underset{\text { PIM-2 with increasing concentration of MWCNTs } \rightarrow}{\rightarrow}$ i
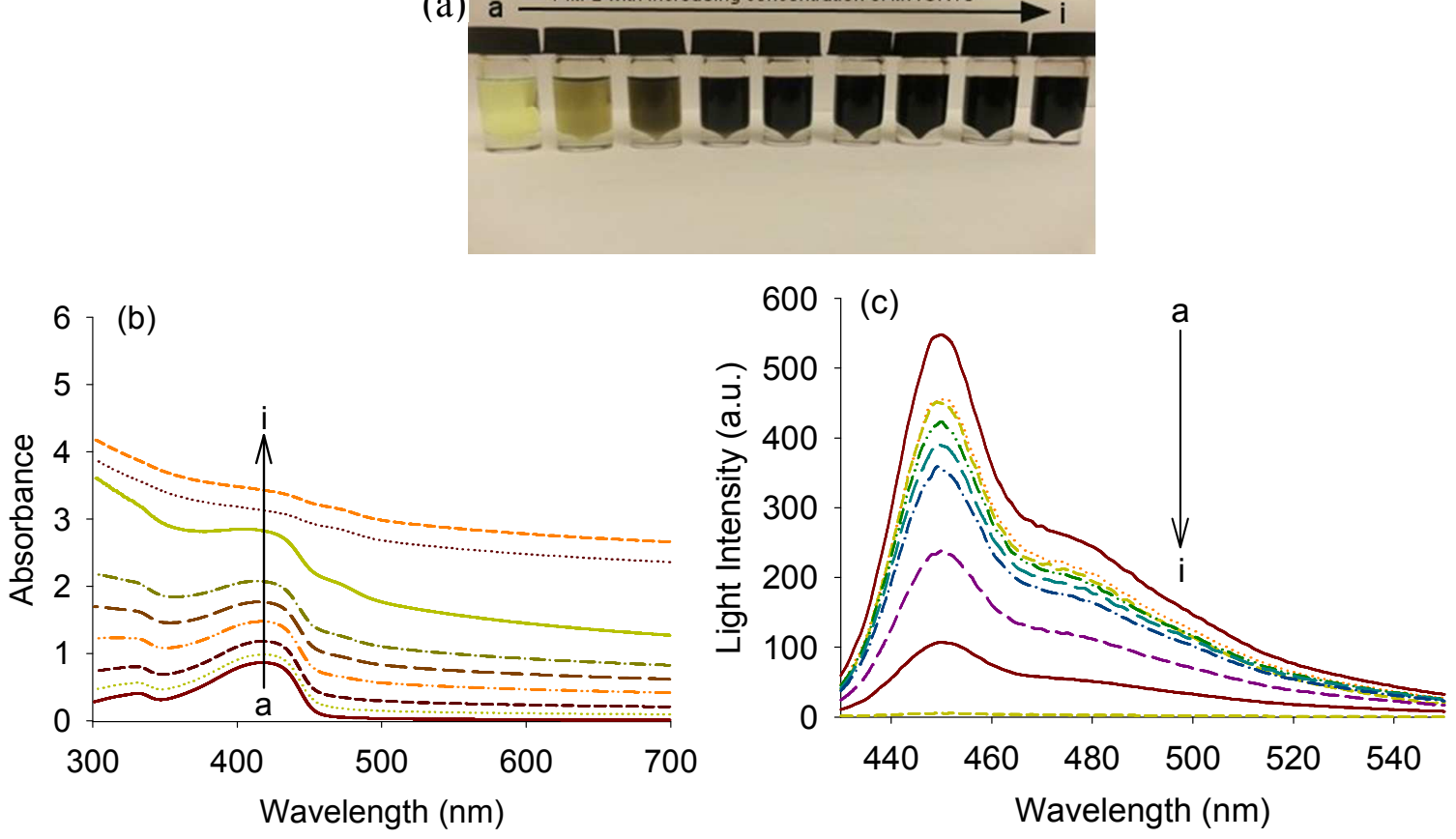

Figure 2 (a) Homogenous dispersions of PIM-2-MWCNTs in DMF (a is $0.11 \mathrm{mg} / \mathrm{ml}$ of PIM-2 and for $\mathrm{b}$ to $\mathrm{f}$ the MWCNT concentrations are $11 \mu \mathrm{g} / \mathrm{ml}, 27.5 \mu \mathrm{g} / \mathrm{ml}, 55 \mu \mathrm{g} / \mathrm{ml}, 82.5 \mu \mathrm{g} / \mathrm{ml}, 110$ $\mu \mathrm{g} / \mathrm{ml}, 220 \mu \mathrm{g} / \mathrm{ml}, 440 \mu \mathrm{g} / \mathrm{ml}, 660 \mu \mathrm{g} / \mathrm{ml}$, this corresponds to the following ratios (wt:wt) MWCNT:PIM-2: 0.1:1, 0.25:1, 0.5:1, 0.75:1, 1:1, 2:1, 4:1, 6:1). The solutions are stable for more than 8 months on the bench at room temperature. (b) UV-visible absorption spectra of PIM2/MWCNTs dispersions in DMF. The concentration of PIM-2 was fixed while the concentration of MWCNTs was increasing. (c) Fluorescence spectra of PIM-2/MWCNTs with increasing concentration of MWCNTs in DMF. Note that for the ratios 4:1 and 6:1, the solution is diluted 5more times. 

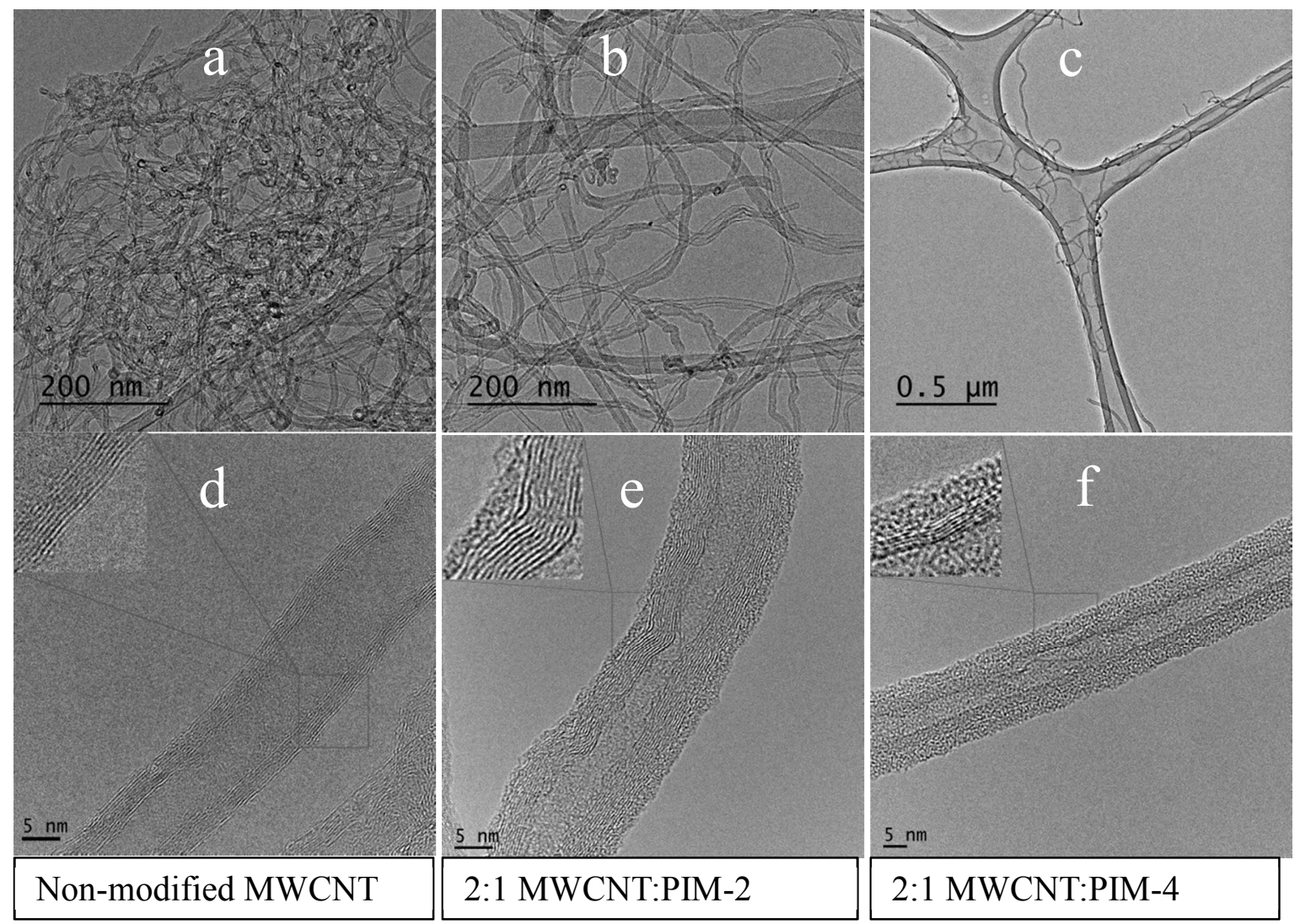

Figure 3 TEM images for (a) Pristine MWCNTs, (b) PIM-2 modified MWCNTs and (c) PIM-4 modified MWCNTs. (d) HR-TEM for pristine MWCNT, the magnified area in the box shows smooth surface of the walls of the nanotube. (e) HR-TEM for the PIM-2 modified MWCNTs; the magnified area in the box shows the walls of the nanotube with a thin layer of the coating polymer on the surface. (f) HR-TEM for the PIM-4 modified MWCNTs; similarly the magnified area in the box shows the walls of the nanotube with a thin layer of the coating polymer on the surface. 
(a)
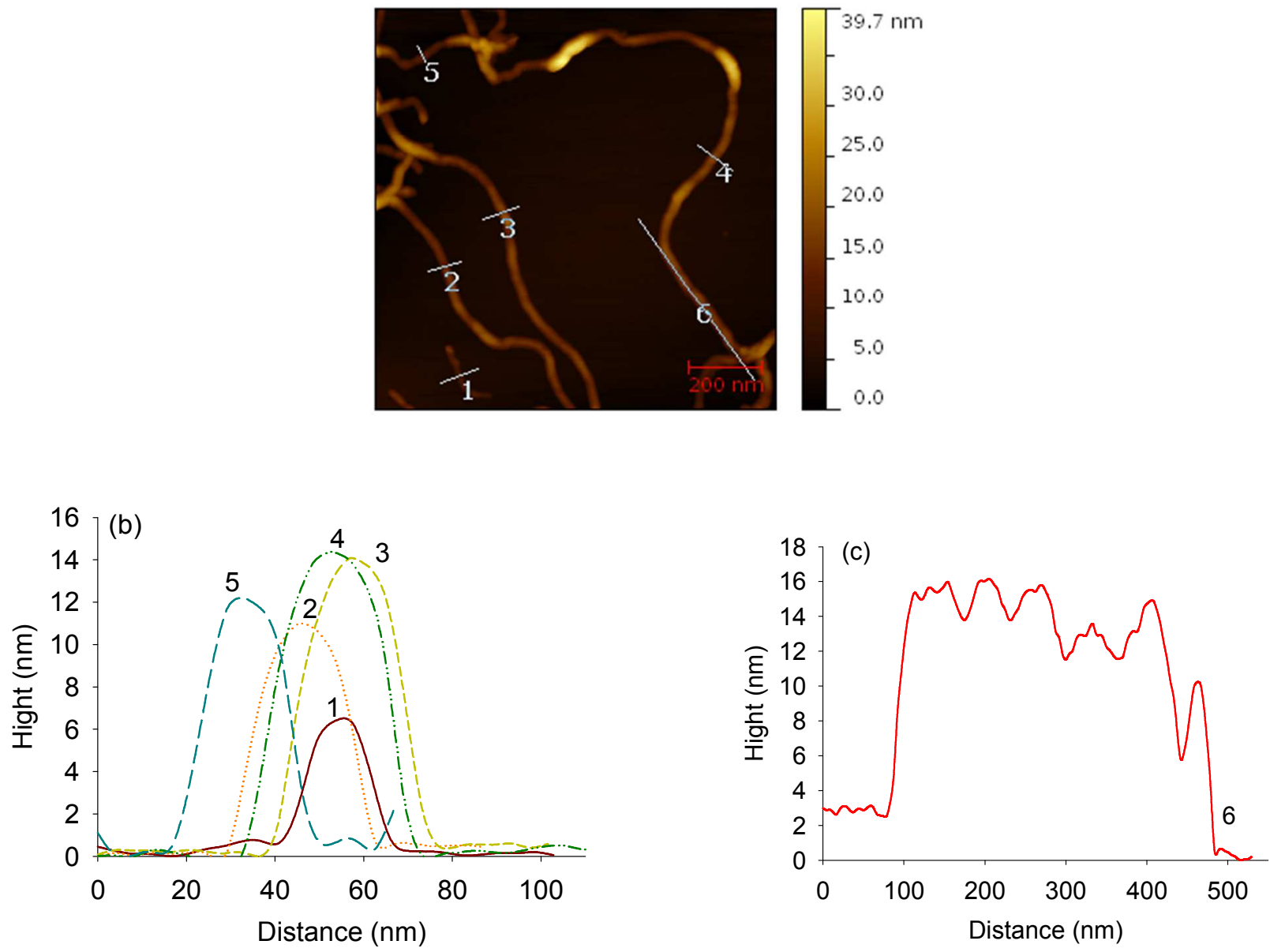

Figure 4 (a) Phase images resulting from intermittent contact mode AFM experiment of 2:1 PIM-2-MWCNT from aqueous suspension on Mica surface. (b) Height profiles of the MWCNTs in the AFM image. (c) Height profile of the modified MWCNT (line 6) in the AFM image. 


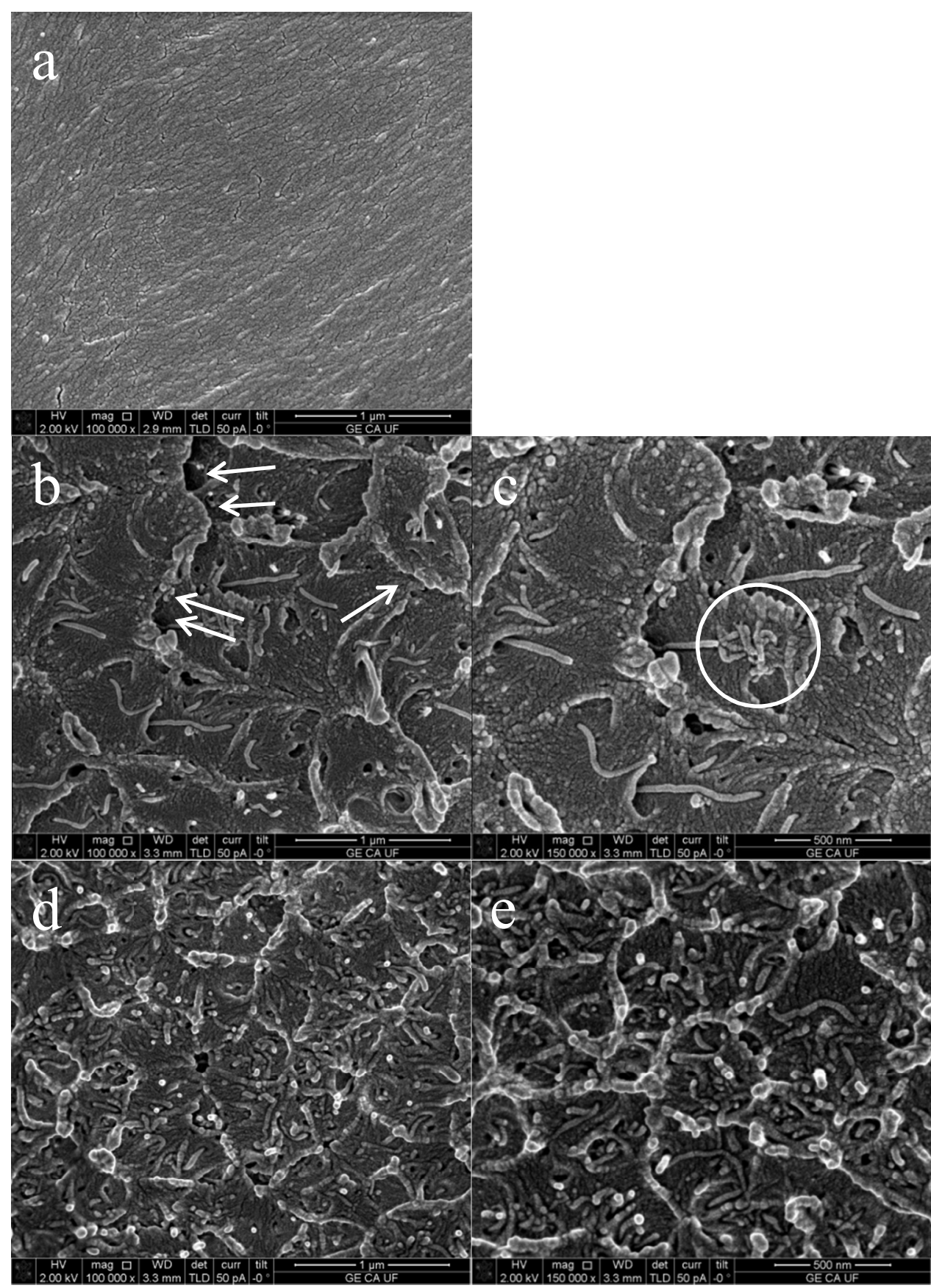

Figure 5 HR-SEM images displaying the architecture of the cryofractured composites. (a) PMMA with $1 \mathrm{wt} \%$ PIM-2 only compared to the whole polymer matrix. (b) PMMA/MWCNTs composite with $1 \mathrm{wt} \%$ pristine MWCNT; The arrows show a clear deformation in the fractured surface of the PMMA composite with observable phase separation. (c) Higher magnification image of (b) showing MWCNTs aggregates; the circle shows MWCNT aggregates in PMMA matrix. (d) PMMA/PIM-2/MWCNTs composite with 1wt\% PIM-2 and $1 \mathrm{wt} \%$ MWCNTs. (e) Higher magnification image of (d) showing homogenous dispersion of the MWCNTs in the PMMA matrix. 


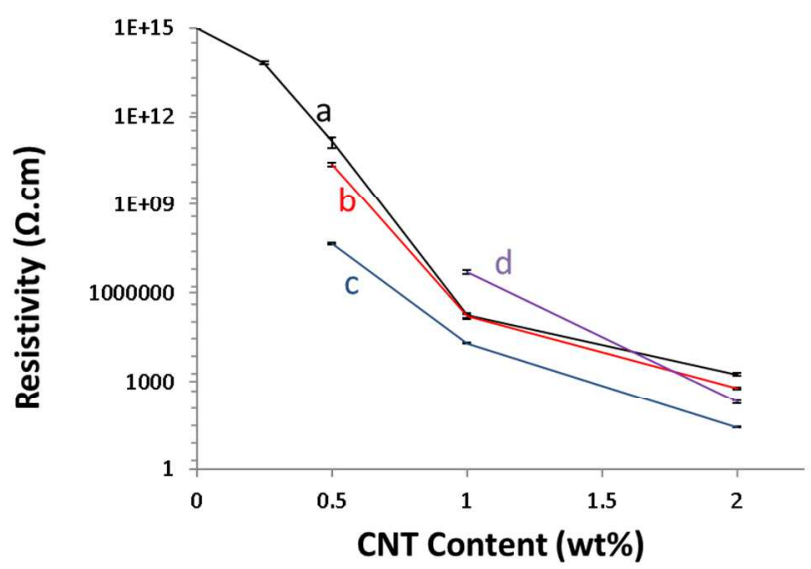

Figure 6 Volume resistivity of PMMA and its composites. (a) Volume resistivity values for composites having 1wt\% PIM-2 (compared to the whole PMMA matrix) and increasing concentrations of MWCNTs. (b) Volume resistivity values for composites having $0.5 \mathrm{wt} \%$ PIM2 and increasing concentrations of MWCNTs. (c) Volume resistivity values for composites having 0.25 wt \% PIM-2 and increasing concentrations of MWCNTs. (d) Volume resistivity values for composites having $0.5 \mathrm{wt} \%$ PIM-4 and increasing concentrations of MWCNTs. All measurements are reproduced several times at room temperature and the average values were plotted accordingly.
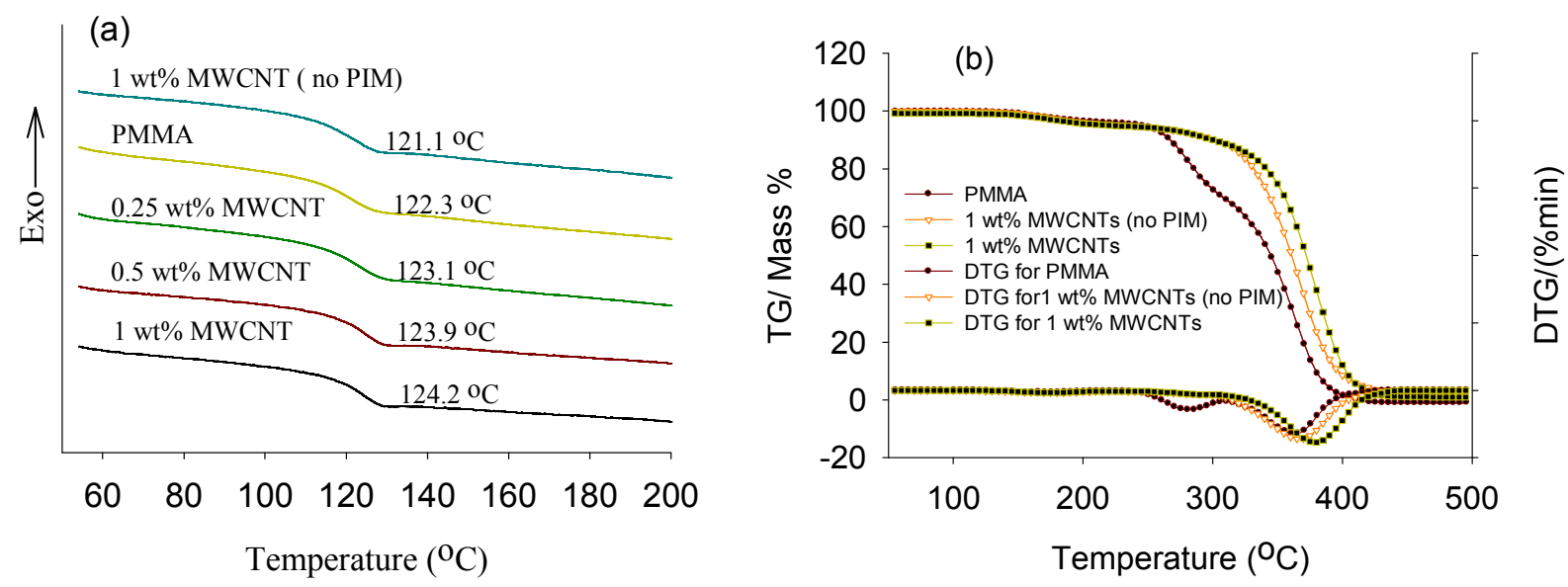

Figure 7 (a) DSC curves of PMMA composite with $1 \mathrm{wt} \%$ pristine MWCNTs, clean PMMA and PMMA composites with $1 \mathrm{wt} \%$ PIM-2 and various concentrations of MWCNTs $(0.25,0.5$ and $1 \mathrm{wt} \%$ of MWCNTs) (b) TGA and DTGA curves of PMMA, PMMA composite with $1 \mathrm{wt} \%$ MWCNTs (no PIM-2), PMMA composite $1 \mathrm{wt} \%$ MWCNT and 1:1 (wt:wt) PIM-2/MWCNT. 
Table 1 Thermal Properties of PMMA and its composites.

\begin{tabular}{|c|c|c|c|c|}
\hline & $\begin{array}{c}\mathrm{T}_{10}{ }^{\mathrm{a}} \\
\left({ }^{\circ} \mathrm{C}\right)\end{array}$ & $\begin{array}{c}\mathrm{T}_{30}{ }^{\mathrm{b}} \\
\left({ }^{\circ} \mathrm{C}\right)\end{array}$ & $\begin{array}{c}\text { Weight loss at } 279{ }^{\circ} \mathrm{C} \\
(\%)\end{array}$ & $\begin{array}{c}\mathrm{T}_{\mathrm{g}} \text { by DSC } \\
\left({ }^{\circ} \mathrm{C}\right)\end{array}$ \\
\hline PMMA & 245 & 300 & 20 & 122 \\
\hline $0.25 \mathrm{wt}^{\circ} \% \mathrm{MWCNTs}^{\mathrm{c}}$ & 285 & 350 & 8.4 & 123 \\
\hline $0.5 \mathrm{wt} \% \mathrm{MWCNTs}^{\mathrm{c}}$ & 285 & 352 & 8.0 & 124 \\
\hline $1 \mathrm{wt}^{\mathrm{o}} \% \mathrm{MWCNTs}^{\mathrm{c}}$ & 295 & 355 & 7.6 & 121 \\
\hline $1 \mathrm{wt} \% \mathrm{MWCNTs}$ (no PIM) & 293 & 345 & 7.6 & 124 \\
\hline
\end{tabular}

${ }^{\mathrm{a}}$ temperature at $10 \mathrm{wt} \%$ weight loss $\left({ }^{\circ} \mathrm{C}\right),{ }^{\mathrm{b}}$ temperature at $30 \mathrm{wt} \%$ weight loss $\left({ }^{\mathrm{o}} \mathrm{C}\right),{ }^{\mathrm{c}} \mathrm{PMMA}$ composites with $1 \mathrm{wt} \%$ PIM-2 compared to PMMA.

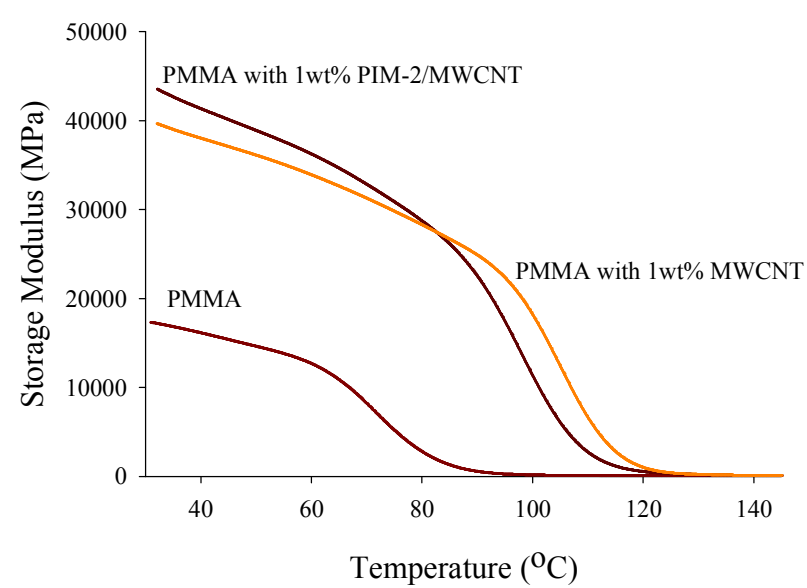

Figure 8 DMA curves for pristine PMMA, PMMA composite with 1 wt $\%$ PIM-2 and 1 wt $\%$ MWCNT (1:1 (wt:wt) PIM-2/MWCNT), PMMA composite with $1 \mathrm{wt} \%$ pristine MWCNTs (no PIM-2). 


\section{TABLE OF CONTENT GRAPHIC}

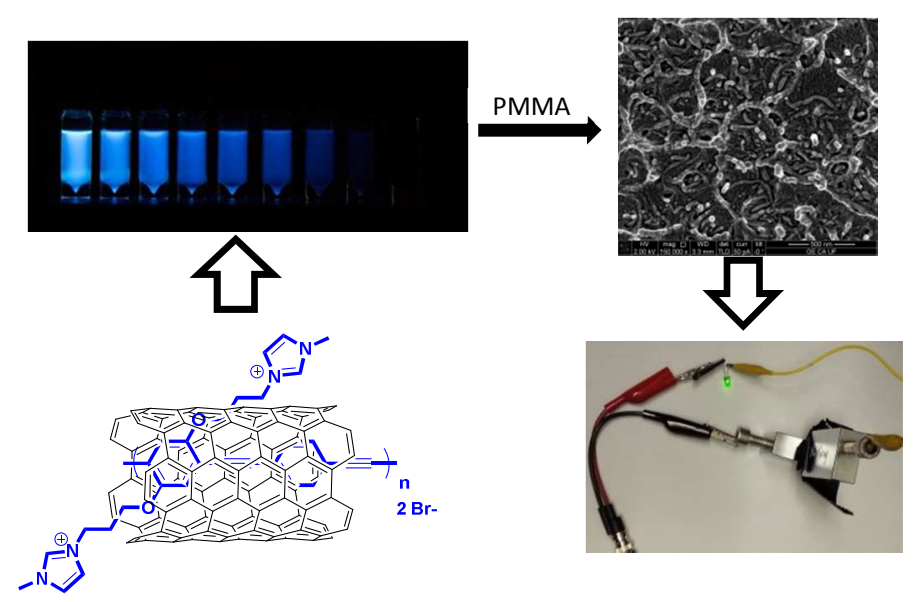

